\title{
Strong convergence of a Halpern-type algorithm for common solutions of fixed point and equilibrium problems
}

\section{Qingnian Zhang*}

\section{"Correspondence:}

wszhangqn@yeah.net

School of Mathematics and

Information Science, North China

University of Water Resources and Electric Power, Zhengzhou, Henan 450011, China

\begin{abstract}
In this article, fixed points of nonexpansive mappings and equilibrium problems based on a Halpern-type algorithm are investigated. Strong convergence theorems for common solutions of the two problems are obtained in the framework of real Hilbert spaces.
\end{abstract}

Keywords: nonexpansive mapping; equilibrium problem; fixed point; variational inequality

\section{Introduction}

The study of equilibrium problems is an important branch of optimization theory and nonlinear functional analysis. Numerous problems in physics, optimization, transportation, signal processing, and economics are reduced to find a solution to equilibrium problems, which cover fixed point problems, variational inequalities, saddle problems, inclusion problems, and so on. A closely related subject of current interest is the problem of finding common elements in the fixed point set of nonlinear operators and in the solution set of monotone variational inequalities; see [1-15] and the references therein. The motivation for this subject is mainly due to its possible applications to mathematical modeling of concrete complex problems. The aim of this paper is to investigate a common element problem based on a Halpern-type algorithm. Strong convergence of the algorithm is obtained in the framework of real Hilbert spaces. The organization of this paper is as follows. In Section 2, we provide some necessary preliminaries. In Section 3, a Halpern-type algorithm is proposed and analyzed. Strong convergence theorems for common solutions of two problems are established in the framework of Hilbert spaces. In Section 4, applications of the main results are provided.

\section{Preliminaries}

Let $H$ be a real Hilbert space with inner product $\langle\cdot, \cdot\rangle$ and norm $\|\cdot\|$. Let $C$ be a nonempty, closed, and convex subset of $H$ and let $\operatorname{Proj}_{C}$ be the metric projection from $H$ onto $C$.

Let $T: C \rightarrow C$ be a mapping. In this paper, we use $F(T)$ to denote the fixed point set of $T$. Recall that $T$ is said to be contractive iff there exists a constant $\alpha \in(0,1)$ such that

$$
\|T x-T y\| \leq \alpha\|x-y\|, \quad \forall x, y \in C .
$$

○ 2014 Zhang; licensee Springer. This is an Open Access article distributed under the terms of the Creative Commons Attribution License (http://creativecommons.org/licenses/by/2.0), which permits unrestricted use, distribution, and reproduction in any medium, provided the original work is properly cited. 
For such a case, $T$ is also said to be $\alpha$-contractive. Recall that $T$ is said to be nonexpansive iff

$$
\|T x-T y\| \leq\|x-y\|, \quad \forall x, y \in C .
$$

It is well known that the fixed point set of nonexpansive mappings is nonempty provided that the subset $C$ is bounded, convex, and closed.

Let $A: C \rightarrow H$ be a mapping. Recall that $A$ is said to be monotone iff

$$
\langle A x-A y, x-y\rangle \geq 0, \quad \forall x, y \in C .
$$

Recall that $A$ is said to be inverse-strongly monotone iff there exists a constant $\alpha>0$ such that

$$
\langle A x-A y, x-y\rangle \geq \alpha\|A x-A y\|^{2}, \quad \forall x, y \in C .
$$

For such a case, $A$ is also said to be $\alpha$-inverse-strongly monotone.

Recall that the classical variational inequality is to find an $x \in C$ such that

$$
\langle A x, y-x\rangle \geq 0, \quad \forall y \in C
$$

In this paper, we use $\operatorname{VI}(C, A)$ to denote the solution set of (2.1). It is well known that $x \in C$ is a solution of the variational inequality (2.1) iff $x$ is a solution of the fixed point equation $P_{C}(I-r A) x=x$, where $r>0$ is a constant.

Recall that a set-valued mapping $M: H \rightrightarrows H$ is said to be monotone iff, for all $x, y \in H$, $f \in M x$ and $g \in M y$ imply $\langle x-y, f-g\rangle>0$. M is maximal iff the graph $\operatorname{Graph}(M)$ of $R$ is not properly contained in the graph of any other monotone mapping. It is well known that a monotone mapping $M$ is maximal if and only if, for any $(x, f) \in H \times H,\langle x-y, f-g\rangle \geq 0$, for all $(y, g) \in \operatorname{Graph}(M)$ implies $f \in R x$.

For a maximal monotone operator $M$ on $H$, and $r>0$, we may define the single-valued resolvent $J_{r}: H \rightarrow D(M)$, where $D(M)$ denotes the domain of $M$. It is known that $J_{r}$ is firmly nonexpansive, and $M^{-1}(0)=F\left(J_{r}\right)$, where $F\left(J_{r}\right):=\left\{x \in D(M): x=J_{r} x\right\}$, and $M^{-1}(0):=$ $\{x \in H: 0 \in M x\}$.

Let $A: C \rightarrow H$ be a monotone mapping, and let $F$ be a bifunction of $C \times C$ into $\mathbb{R}$, where $\mathbb{R}$ denotes the set of real numbers. We consider the following generalized equilibrium problem:

$$
\text { Find } x \in C \text { such that } F(x, y)+\langle A x, y-x\rangle \geq 0, \quad \forall y \in C \text {. }
$$

In this paper, we use $\operatorname{EP}(F, A)$ to denote the solution set of the generalized equilibrium problem (2.2).

Next, we give some special cases of the generalized equilibrium problem (2.2).

(I) If $F \equiv 0$, then problem (2.2) is reduced to the classical variational inequality (2.1).

(II) If $A \equiv 0$, the zero mapping, then problem (2.2) is reduced to the following equilibrium problem:

$$
\text { Find } x \in C \text { such that } F(x, y) \geq 0, \quad \forall y \in C \text {. }
$$

In this paper, we use $\operatorname{EP}(F)$ to denote the solution set of the equilibrium problem (2.3). 
To study the equilibrium problems, we may assume that $F$ satisfies the following conditions:

(A1) $F(x, x)=0$ for all $x \in C$;

(A2) $F$ is monotone, i.e., $F(x, y)+F(y, x) \leq 0$ for all $x, y \in C$;

(A3) for each $x, y, z \in C$,

$$
\limsup _{t \downarrow 0} F(t z+(1-t) x, y) \leq F(x, y)
$$

(A4) for each $x \in C, y \mapsto F(x, y)$ is convex and weakly lower semi-continuous.

Recently, many authors have studied fixed point problems of nonexpansive mappings and solution problems of the equilibrium problems (2.2) and (2.3); for more details, see [16-25] and the references therein. In this paper, motivated and inspired by the research going on in this direction, we consider common element problems based on a mean iterative process. Strong convergence of the iterative process is obtained in the framework of real Hilbert spaces. The results presented in this paper improve and extend the corresponding results in Hao [1], Qin et al. [24], Chang et al. [25].

In order to prove our main results, we need the following lemmas.

Lemma 2.1 [26] Assume that $\left\{\alpha_{n}\right\}$ is a sequence of nonnegative real numbers such that

$$
\alpha_{n+1} \leq\left(1-\gamma_{n}\right) \alpha_{n}+\delta_{n}
$$

where $\left\{\gamma_{n}\right\}$ is a sequence in $(0,1)$ and $\left\{\delta_{n}\right\}$ is a sequence such that

(1) $\sum_{n=1}^{\infty} \gamma_{n}=\infty$;

(2) $\lim \sup _{n \rightarrow \infty} \delta_{n} / \gamma_{n} \leq 0$ or $\sum_{n=1}^{\infty}\left|\delta_{n}\right|<\infty$.

Then $\lim _{n \rightarrow \infty} \alpha_{n}=0$.

Lemma 2.2 [27] Let $F: C \times C \rightarrow \mathbb{R}$ be a bifunction satisfying (A1)-(A4). Then, for any $r>0$ and $x \in H$, there exists $z \in C$ such that

$$
F(z, y)+\frac{1}{r}\langle y-z, z-x\rangle \geq 0, \quad \forall y \in C .
$$

Define a mapping $T_{r}: H \rightarrow C$ as follows:

$$
T_{r} x=\left\{z \in C: F(z, y)+\frac{1}{r}\langle y-z, z-x\rangle \geq 0, \forall y \in C\right\}, \quad x \in H,
$$

then the following conclusions hold.

(1) $T_{r}$ is single-valued;

(2) $T_{r}$ is firmly nonexpansive, i.e., for any $x, y \in H$,

$$
\left\|T_{r} x-T_{r} y\right\|^{2} \leq\left\langle T_{r} x-T_{r} y, x-y\right\rangle
$$

(3) $F\left(T_{r}\right)=\mathrm{EP}(F)$;

(4) $\mathrm{EP}(F)$ is closed and convex.

Let $\left\{S_{i}: C \rightarrow C\right\}$ be a family of infinitely nonexpansive mappings and $\left\{\gamma_{i}\right\}$ be a nonnegative real sequence with $0 \leq \gamma_{i}<1, \forall i \geq 1$. For $n \geq 1$ define a mapping $W_{n}: C \rightarrow C$ as 
follows:

$$
\begin{aligned}
& U_{n, n+1}=I, \\
& U_{n, n}=\gamma_{n} S_{n} U_{n, n+1}+\left(1-\gamma_{n}\right) I, \\
& U_{n, n-1}=\gamma_{n-1} S_{n-1} U_{n, n}+\left(1-\gamma_{n-1}\right) I, \\
& \vdots \\
& U_{n, k}=\gamma_{k} S_{k} U_{n, k+1}+\left(1-\gamma_{k}\right) I, \\
& U_{n, k-1}=\gamma_{k-1} S_{k-1} U_{n, k}+\left(1-\gamma_{k-1}\right) I, \\
& \vdots \\
& U_{n, 2}=\gamma_{2} S_{2} U_{n, 3}+\left(1-\gamma_{2}\right) I, \\
& W_{n}=U_{n, 1}=\gamma_{1} S_{1} U_{n, 2}+\left(1-\gamma_{1}\right) I .
\end{aligned}
$$

Such a mapping $W_{n}$ is nonexpansive from $C$ to $C$ and it is called a $W$-mapping generated by $S_{n}, S_{n-1}, \ldots, S_{1}$ and $\gamma_{n}, \gamma_{n-1}, \ldots, \gamma_{1}$; see [28] and the references therein.

Lemma 2.3 [28] Let $\left\{S_{i}: C \rightarrow C\right\}$ be a family of infinitely nonexpansive mappings with a nonempty common fixed point set and let $\left\{\gamma_{i}\right\}$ be a real sequence such that $0<\gamma_{i} \leq l<1$, where $l$ is some real number, $\forall i \geq 1$. Then

(1) $W_{n}$ is nonexpansive and $F\left(W_{n}\right)=\bigcap_{i=1}^{\infty} F\left(S_{i}\right)$, for each $n \geq 1$;

(2) for each $x \in C$ and for each positive integer $k$, the limit $\lim _{n \rightarrow \infty} U_{n, k}$ exists;

(3) the mapping $W: C \rightarrow C$ defined by

$$
W x:=\lim _{n \rightarrow \infty} W_{n} x=\lim _{n \rightarrow \infty} U_{n, 1} x, \quad x \in C,
$$

is a nonexpansive mapping satisfying $F(W)=\bigcap_{i=1}^{\infty} F\left(S_{i}\right)$ and it is called the $W$-mapping generated by $S_{1}, S_{2}, \ldots$ and $\gamma_{1}, \gamma_{2}, \ldots$.

Lemma 2.4 [29] Let $B: C \rightarrow H$ be a mapping and let $M: H \rightrightarrows H$ be a maximal monotone operator. Then $F\left(J_{r}(I-r B)\right)=(B+M)^{-1}(0)$, where $r$ is some positive real number.

Lemma 2.5 [25] Let $\left\{S_{i}: C \rightarrow C\right\}$ be a family of infinitely nonexpansive mappings with a nonempty common fixed point set and let $\left\{\gamma_{i}\right\}$ be a real sequence such that $0<\gamma_{i} \leq l<1$, $\forall i \geq 1$. If $K$ is any bounded subset of $C$, then

$$
\lim _{n \rightarrow \infty} \sup _{x \in K}\left\|W x-W_{n} x\right\|=0 .
$$

Throughout this paper, we always assume that $0<\gamma_{i} \leq l<1, \forall i \geq 1$.

Lemma 2.6 [30] Let $A: C \rightarrow H$ a Lipschitz monotone mapping and let $N_{C} x$ be the normal cone to $C$ at $x \in C$; that is, $N_{C} x=\{y \in H:\langle x-u, y\rangle, \forall u \in C\}$. Define

$$
W x= \begin{cases}A x+N_{C} x, & x \in C, \\ \varnothing & x \notin C .\end{cases}
$$

Then $W$ is maximal monotone and $0 \in W x$ if and only if $x \in \mathrm{VI}(C, A)$. 
Lemma 2.7 [31] Let $\left\{x_{n}\right\}$ and $\left\{y_{n}\right\}$ be bounded sequences in $H$ and let $\left\{\beta_{n}\right\}$ be a sequence in $(0,1)$ with $0<\liminf _{n \rightarrow \infty} \beta_{n} \leq \limsup _{n \rightarrow \infty} \beta_{n}<1$. Suppose that $x_{n+1}=\left(1-\beta_{n}\right) y_{n}+\beta_{n} x_{n}$ for all $n \geq 0$ and

$$
\limsup _{n \rightarrow \infty}\left(\left\|y_{n+1}-y_{n}\right\|-\left\|x_{n+1}-x_{n}\right\|\right) \leq 0 .
$$

Then $\lim _{n \rightarrow \infty}\left\|y_{n}-x_{n}\right\|=0$.

\section{Main results}

Theorem 3.1 Let $C$ be a nonempty closed convex subset of a Hilbert space $H$ and let $F$ be a bifunction from $C \times C$ to $\mathbb{R}$ which satisfies (A1)-(A4). Let $A: C \rightarrow H$ be an $\alpha$-inversestrongly monotone mapping and let $B: C \rightarrow H$ be a $\beta$-inverse-strongly monotone mapping. Let $M: H \rightrightarrows H$ be a maximal monotone operator. Let $\left\{S_{i}: C \rightarrow C\right\}$ be a family of infinitely nonexpansive mappings. Assume that $F:=\bigcap_{i=1}^{\infty} F\left(S_{i}\right) \cap \mathrm{EP}(F, B) \cap(A+M)^{-1}(0) \neq \emptyset$. Let $f: C \rightarrow C$ be a $\kappa$-contraction. Let $\left\{x_{n}\right\}$ be a sequence generated by the process: $x_{1} \in C$ and

$$
\left\{\begin{array}{l}
F\left(u_{n}, y\right)+\left\langle B x_{n}, y-u_{n}\right\rangle+\frac{1}{s_{n}}\left\langle y-u_{n}, u_{n}-x_{n}\right\rangle \geq 0, \quad \forall y \in C, \\
x_{n+1}=\alpha_{n} f\left(x_{n}\right)+\beta_{n} x_{n}+\gamma_{n} W_{n} \operatorname{Proj}_{C} J_{r_{n}}\left(u_{n}-r_{n} A u_{n}\right), \quad \forall n \geq 1,
\end{array}\right.
$$

where $\left\{W_{n}: C \rightarrow C\right\}$ is the sequence generated in (2.4), $\left\{\alpha_{n}\right\},\left\{\beta_{n}\right\}$, and $\left\{\gamma_{n}\right\}$ are sequences in $(0,1)$ such that $\alpha_{n}+\beta_{n}+\gamma_{n}=1$ and $\left\{r_{n}\right\}$ and $\left\{s_{n}\right\}$ are positive number sequences. Assume that the above control sequences satisfy the following restrictions:

(a) $0<r \leq r_{n} \leq r^{\prime}<2 \alpha, 0<r^{\prime \prime} \leq s_{n} \leq r^{\prime \prime \prime}<2 \beta$;

(b) $\lim _{n \rightarrow \infty} \alpha_{n}=0$ and $\sum_{n=1}^{\infty} \alpha_{n}=\infty$;

(c) $0<\liminf _{n \rightarrow \infty} \beta_{n} \leq \limsup _{n \rightarrow \infty} \beta_{n}<1$;

(d) $\lim _{n \rightarrow \infty}\left|s_{n}-s_{n+1}\right|=\lim _{n \rightarrow \infty}\left|r_{n}-r_{n+1}\right|=0$.

Then the sequence $\left\{x_{n}\right\}$ converges strongly to $\bar{x}=\operatorname{Proj}_{F} f(\bar{x})$.

Proof First, we show that $I-r_{n} A$ is nonexpansive. For $\forall x, y \in C$, we have

$$
\begin{aligned}
\| & \left(I-r_{n} A\right) x-\left(I-r_{n} A\right) y \|^{2} \\
& =\|x-y\|^{2}-2 r_{n}\langle x-y, A x-A y\rangle+r_{n}^{2}\|A x-A y\|^{2} \\
& \leq\|x-y\|^{2}-2 r_{n} \alpha\|A x-A y\|^{2}+r_{n}^{2}\|A x-A y\|^{2} \\
& =\|x-y\|^{2}+r_{n}\left(r_{n}-2 \alpha\right)\|A x-A y\|^{2} .
\end{aligned}
$$

Using restriction (a), we have $I-r_{n} A$ is nonexpansive, so is $I-s_{n} B$. Fix $x^{*} \in F$. It follows that $\left\|u_{n}-x^{*}\right\| \leq\left\|\left(I-s_{n} B\right) x_{n}-\left(I-s_{n} B\right) x^{*}\right\| \leq\left\|x_{n}-x^{*}\right\|$. Putting $y_{n}=J_{r_{n}}\left(u_{n}-r_{n} A u_{n}\right)$, one finds that $\left\|y_{n}-x^{*}\right\| \leq\left\|u_{n}-x^{*}\right\| \leq\left\|x_{n}-x^{*}\right\|$. It follows that

$$
\begin{aligned}
\left\|x_{n+1}-x^{*}\right\| & \leq \alpha_{n}\left\|f\left(x_{n}\right)-x^{*}\right\|+\beta_{n}\left\|x_{n}-x^{*}\right\|+\gamma_{n}\left\|W_{n} \operatorname{Proj}_{C} y_{n}-x^{*}\right\| \\
& \leq \alpha_{n}\left\|f\left(x_{n}\right)-f\left(x^{*}\right)\right\|+\alpha_{n}\left\|f\left(x^{*}\right)-x^{*}\right\|+\beta_{n}\left\|x_{n}-x^{*}\right\|+\gamma_{n}\left\|y_{n}-x^{*}\right\| \\
& \leq\left(1-\alpha_{n}(1-\kappa)\right)\left\|x_{n}-x^{*}\right\|+\alpha_{n}\left\|f\left(x^{*}\right)-x^{*}\right\| .
\end{aligned}
$$

Hence, we have $\left\|x_{n}-x^{*}\right\| \leq \max \left\{\left\|x_{1}-x^{*}\right\|, \frac{\left\|f\left(x^{*}\right)-x^{*}\right\|}{1-\alpha}\right\}$. This yields the result that $\left\{x_{n}\right\}$ is bounded. Therefore, both $\left\{y_{n}\right\}$ and $\left\{u_{n}\right\}$ are also bounded. Next, without loss of generality, 
we assume that there exists a bounded set $K \subset C$ such that $x_{n}, y_{n}, u_{n} \in K$. Notice that $F\left(u_{n+1}, y\right)+\frac{1}{s_{n+1}}\left\langle y-u_{n+1}, u_{n+1}-\left(I-s_{n+1} B\right) x_{n+1}\right\rangle \geq 0, \forall y \in C$, and $F\left(u_{n}, y\right)+\frac{1}{s_{n}}\left\langle y-u_{n}, u_{n}-\right.$ $\left.\left(I-s_{n} B\right) x_{n}\right\rangle \geq 0, \forall y \in C$. It follows that

$$
\left\langle u_{n+1}-u_{n}, \frac{u_{n}-\left(I-s_{n} B\right) x_{n}}{s_{n}}-\frac{u_{n+1}-\left(I-s_{n+1} B\right) x_{n+1}}{s_{n+1}}\right\rangle \geq 0 .
$$

Hence, we have

$$
\begin{aligned}
\left\|u_{n+1}-u_{n}\right\|^{2} \leq & \left\langle u_{n+1}-u_{n},\left(I-s_{n+1} B\right) x_{n+1}-\left(I-s_{n} B\right) x_{n}\right. \\
& \left.+\left(1-\frac{s_{n}}{s_{n+1}}\right)\left(u_{n+1}-\left(I-s_{n+1} B\right) x_{n+1}\right)\right\rangle \\
\leq & \left\|u_{n+1}-u_{n}\right\|\left(\left\|\left(I-s_{n+1} B\right) x_{n+1}-\left(I-s_{n} B\right) x_{n}\right\|\right. \\
& \left.+\left|1-\frac{s_{n}}{s_{n+1}}\right|\left\|u_{n+1}-\left(I-s_{n+1} B\right) x_{n+1}\right\|\right) .
\end{aligned}
$$

This yields the result that

$$
\begin{aligned}
\left\|u_{n+1}-u_{n}\right\| \leq & \left\|\left(I-s_{n+1} B\right) x_{n+1}-\left(I-s_{n} B\right) x_{n}\right\| \\
& +\frac{\left|s_{n+1}-s_{n}\right|}{s_{n+1}}\left\|u_{n+1}-\left(I-s_{n+1} B\right) x_{n+1}\right\| \\
= & \left\|\left(I-s_{n+1} B\right) x_{n+1}-\left(I-s_{n+1} B\right) x_{n}+\left(I-s_{n+1} B\right) x_{n}-\left(I-s_{n} B\right) x_{n}\right\| \\
& \quad+\frac{\left|s_{n+1}-s_{n}\right|}{s_{n+1}}\left\|u_{n+1}-\left(I-s_{n+1} B\right) x_{n+1}\right\| \\
\leq & \left\|x_{n+1}-x_{n}\right\|+\left|s_{n+1}-s_{n}\right| M_{1},
\end{aligned}
$$

where $M_{1}$ is an appropriate constant such that

$$
M_{1}=\sup _{n \geq 1}\left\{\left\|B x_{n}\right\|+\frac{\left\|u_{n+1}-\left(I-s_{n+1} B\right) x_{n+1}\right\|}{\bar{a}}\right\} .
$$

Since $J_{r_{n}}$ is firmly nonexpansive, one sees that

$$
\begin{aligned}
& \left\|y_{n+1}-y_{n}\right\| \\
& \quad=\left\|J_{r_{n}}\left(u_{n+1}-r_{n+1} A u_{n+1}\right)-J_{r_{n}}\left(u_{n}-r_{n} A u_{n}\right)\right\| \\
& \quad \leq\left\|u_{n+1}-r_{n+1} A u_{n+1}-\left(u_{n}-r_{n} A u_{n}\right)\right\| \\
& \quad=\left\|\left(I-r_{n+1} A\right) u_{n+1}-\left(I-r_{n+1} A\right) u_{n}+\left(r_{n}-r_{n+1}\right) A u_{n}\right\| \\
& \quad \leq\left\|u_{n+1}-u_{n}\right\|+\left|r_{n}-r_{n+1}\right|\left\|A u_{n}\right\| .
\end{aligned}
$$

Substituting (3.1) into (3.2), one finds that

$$
\left\|y_{n+1}-y_{n}\right\| \leq\left\|x_{n+1}-x_{n}\right\|+\left(\left|s_{n+1}-s_{n}\right|+\left|r_{n}-r_{n+1}\right|\right) M_{2},
$$


where $M_{2}$ is an appropriate constant such that $M_{2}=\max \left\{\sup _{n \geq 1}\left\{\left\|A u_{n}\right\|\right\}, M_{1}\right\}$. On the other hand, one has

$$
\begin{aligned}
\| & W_{n+1} \operatorname{Proj}_{C} y_{n+1}-W_{n} \operatorname{Proj}_{C} y_{n} \| \\
& \leq\left\|W_{n+1} y_{n+1}-W_{n} y_{n}\right\| \\
& \leq\left\|W_{n+1} y_{n+1}-W y_{n+1}\right\|+\left\|W y_{n+1}-W y_{n}\right\|+\left\|W y_{n}-W_{n} y_{n}\right\| \\
& \leq \sup _{x \in K}\left\{\left\|W_{n+1} x-W x\right\|+\left\|W x-W_{n} x\right\|\right\}+\left\|y_{n+1}-y_{n}\right\|,
\end{aligned}
$$

where $K$ is the bounded subset of $C$ defined above. Combining (3.3) with (3.4), one finds

$$
\begin{aligned}
& \left\|W_{n+1} \operatorname{Proj}_{C} y_{n+1}-W_{n} \operatorname{Proj}_{C} y_{n}\right\| \\
& \leq \sup _{x \in K}\left\{\left\|W_{n+1} x-W x\right\|+\left\|W x-W_{n} x\right\|\right\}+\left\|x_{n+1}-x_{n}\right\| \\
& \quad+\left(\left|r_{n+1}-r_{n}\right|+\left|s_{n}-s_{n+1}\right|\right) M_{2} .
\end{aligned}
$$

Letting $x_{n+1}=\left(1-\beta_{n}\right) z_{n}+\beta_{n} x_{n}$ we see that

$$
\begin{aligned}
\left\|z_{n+1}-z_{n}\right\| \leq & \frac{\alpha_{n+1}}{1-\beta_{n+1}}\left\|f\left(x_{n+1}\right)-W_{n+1} y_{n+1}\right\|+\frac{\alpha_{n}}{1-\beta_{n}}\left\|f\left(x_{n}\right)-W_{n} y_{n}\right\| \\
& +\left\|W_{n+1} \operatorname{Proj}_{C} y_{n+1}-W_{n} \operatorname{Proj}_{C} y_{n}\right\| .
\end{aligned}
$$

Substituting (3.5) into (3.6), we see that

$$
\begin{aligned}
& \left\|z_{n+1}-z_{n}\right\|-\left\|x_{n+1}-x_{n}\right\| \\
& \leq \frac{\alpha_{n+1}}{1-\beta_{n+1}}\left\|f\left(x_{n+1}\right)-W_{n+1} y_{n+1}\right\|+\frac{\alpha_{n}}{1-\beta_{n}}\left\|f\left(x_{n}\right)-W_{n} y_{n}\right\| \\
& \quad+\sup _{x \in K}\left\{\left\|W_{n+1} x-W x\right\|+\left\|W x-W_{n} x\right\|\right\} \\
& \quad+\left(\left|r_{n+1}-r_{n}\right|+\left|s_{n}-s_{n+1}\right|\right) M_{2} .
\end{aligned}
$$

It follows from restrictions (a), (c), and (d) that $\lim _{\sup } \rightarrow \infty\left(\left\|z_{n+1}-z_{n}\right\|-\left\|x_{n+1}-x_{n}\right\|\right) \leq 0$. Using Lemma 2.7, we find that $\lim _{n \rightarrow \infty}\left\|z_{n}-x_{n}\right\|=0$. It follows that

$$
\lim _{n \rightarrow \infty}\left\|x_{n+1}-x_{n}\right\|=0
$$

For any $x^{*} \in F$, we see that

$$
\begin{aligned}
\left\|x_{n+1}-x^{*}\right\|^{2} & \leq \alpha_{n}\left\|f\left(x_{n}\right)-x^{*}\right\|^{2}+\beta_{n}\left\|x_{n}-x^{*}\right\|^{2}+\gamma_{n}\left\|W_{n} \operatorname{Proj}_{C} y_{n}-x^{*}\right\|^{2} \\
& \leq \alpha_{n}\left\|f\left(x_{n}\right)-x^{*}\right\|^{2}+\beta_{n}\left\|x_{n}-x^{*}\right\|^{2}+\gamma_{n}\left\|y_{n}-x^{*}\right\|^{2} .
\end{aligned}
$$

Since

$$
\begin{aligned}
\left\|y_{n}-x^{*}\right\|^{2} & =\left\|J_{r_{n}}\left(u_{n}-r_{n} A u_{n}\right)-x^{*}\right\|^{2} \\
& \leq\left\|\left(I-r_{n} A\right) u_{n}-\left(I-r_{n} A\right) x^{*}\right\|^{2}
\end{aligned}
$$




$$
\begin{aligned}
& =\left\|u_{n}-x^{*}\right\|^{2}-2 r_{n}\left\langle u_{n}-x^{*}, A u_{n}-A x^{*}\right\rangle+r_{n}^{2}\left\|A u_{n}-A x^{*}\right\|^{2} \\
& \leq\left\|x_{n}-x^{*}\right\|^{2}+r_{n}\left(r_{n}-2 \alpha\right)\left\|A u_{n}-A x^{*}\right\|^{2}
\end{aligned}
$$

we find from (3.8) that

$$
\lim _{n \rightarrow \infty}\left\|A u_{n}-A x^{*}\right\|=0
$$

It also follows from (3.8) that

$$
\begin{aligned}
\left\|x_{n+1}-x^{*}\right\|^{2} \leq & \alpha_{n}\left\|f\left(x_{n}\right)-x^{*}\right\|^{2}+\beta_{n}\left\|x_{n}-x^{*}\right\|^{2}+\gamma_{n}\left\|x_{n}-x^{*}-s_{n}\left(B x_{n}-B x^{*}\right)\right\|^{2} \\
\leq & \alpha_{n}\left\|f\left(x_{n}\right)-x^{*}\right\|^{2}+\beta_{n}\left\|x_{n}-x^{*}\right\|^{2} \\
& +\gamma_{n}\left(\left\|x_{n}-x^{*}\right\|^{2}+s_{n}^{2}\left\|B x_{n}-B x^{*}\right\|^{2}-2 s_{n}\left\langle B x_{n}-B x^{*}, x_{n}-x^{*}\right\rangle\right) \\
\leq & \alpha_{n}\left\|f\left(x_{n}\right)-x^{*}\right\|^{2}+\beta_{n}\left\|x_{n}-x^{*}\right\|^{2}+\gamma_{n}\left\|x_{n}-x^{*}\right\|^{2} \\
& -s_{n} \gamma_{n}\left(2 \beta-s_{n}\right)\left\|B x_{n}-B x^{*}\right\|^{2} .
\end{aligned}
$$

Using (3.7), one arrives at

$$
\lim _{n \rightarrow \infty}\left\|B x_{n}-B x^{*}\right\|=0
$$

Since $T_{s_{n}}$ is firmly nonexpansive, we find that

$$
\begin{aligned}
\left\|u_{n}-x^{*}\right\|^{2} \leq & \left\langle\left(I-s_{n} B\right) x_{n}-\left(I-s_{n} B\right) x^{*}, u_{n}-x^{*}\right\rangle \\
\leq & \frac{1}{2}\left(\left\|x_{n}-x^{*}\right\|^{2}+\left\|u_{n}-x^{*}\right\|^{2}-\left\|x_{n}-u_{n}\right\|^{2}-s_{n}^{2}\left\|B x_{n}-B x^{*}\right\|^{2}\right. \\
& \left.+2 s_{n}\left\langle B x_{n}-B x^{*}, x_{n}-u_{n}\right\rangle\right),
\end{aligned}
$$

which implies that $\left\|u_{n}-x^{*}\right\|^{2} \leq\left\|x_{n}-x^{*}\right\|^{2}-\left\|x_{n}-u_{n}\right\|^{2}+2 s_{n}\left\|B x_{n}-B x^{*}\right\|\left\|x_{n}-u_{n}\right\|$. Hence

$$
\begin{aligned}
\gamma_{n}\left\|x_{n}-u_{n}\right\|^{2} \leq & \alpha_{n}\left\|f\left(x_{n}\right)-x^{*}\right\|^{2}+\left(\left\|x_{n}-x^{*}\right\|+\left\|x_{n+1}-x^{*}\right\|\right)\left\|x_{n}-x_{n+1}\right\| \\
& +2 s_{n}\left\|B x_{n}-B x^{*}\right\|\left\|x_{n}-u_{n}\right\| .
\end{aligned}
$$

Using (3.7) and (3.10), one has

$$
\lim _{n \rightarrow \infty}\left\|x_{n}-u_{n}\right\|=0
$$

Similarly, one also has

$$
\lim _{n \rightarrow \infty}\left\|y_{n}-u_{n}\right\|=0
$$

Since

$$
\left\|W_{n} y_{n}-y_{n}\right\| \leq\left\|y_{n}-u_{n}\right\|+\left\|u_{n}-x_{n}\right\|+\left\|x_{n}-W_{n} y_{n}\right\|
$$


we find from (3.11) and (3.12) that

$$
\lim _{n \rightarrow \infty}\left\|W_{n} y_{n}-y_{n}\right\|=0
$$

Now, we are in a position to show $\lim \sup _{n \rightarrow \infty}\left\langle f(\bar{x})-\bar{x}, x_{n}-z\right\rangle \leq 0$, where $\bar{x}=\operatorname{Proj}_{F} f(\bar{x})$. To prove this, we choose a subsequence $\left\{x_{n_{i}}\right\}$ of $\left\{x_{n}\right\}$ such that

$$
\limsup _{n \rightarrow \infty}\left\langle f(\bar{x})-\bar{x}, x_{n}-\bar{x}\right\rangle=\lim _{i \rightarrow \infty}\left\langle f(\bar{x})-\bar{x}, x_{n_{i}}-\bar{x}\right\rangle .
$$

Since $\left\{x_{n_{i}}\right\}$ is bounded, without loss of generality, we may assume that $x_{n_{i}} \rightarrow q$. Using (3.11) and (3.12), we have $\lim _{n \rightarrow \infty}\left\|x_{n}-y_{n}\right\|=0$. Therefore, we see that $y_{n_{i}} \rightarrow q$. Now, we are in a position to prove $q \in(A+M)^{-1}(0)$. Notice that $\frac{u_{n}-y_{n}}{r_{n}}-A u_{n} \in M y_{n}$. Let $\mu \in M v$. Since $M$ is monotone, we find that $\left\langle\frac{u_{n}-y_{n}}{r_{n}}-A u_{n}-\mu, y_{n}-v\right\rangle \geq 0$. This implies that $\langle-A q-\mu, q-v\rangle \geq 0$. This implies that $-A q \in M q$, that is, $q \in(A+M)^{-1}(0)$. Next, we show that $q \in \operatorname{EP}(F, B)$. Since $u_{n}=T_{s_{n}}\left(I-s_{n} B\right) x_{n}$, we find from (A2) that

$$
\left\langle B x_{n_{i}}, y-u_{n_{i}}\right\rangle+\left\langle y-u_{n_{i}}, \frac{u_{n_{i}}-x_{n_{i}}}{s_{n_{i}}}\right\rangle \geq F\left(y, u_{n_{i}}\right), \quad \forall y \in C .
$$

Putting $y_{t}=t y+(1-t) q$ for any $t \in(0,1]$ and $y \in C$, we see that $y_{t} \in C$. Using (3.15), we find that

$$
\begin{aligned}
& \left\langle y_{t}-u_{n_{i}}, B y_{t}\right\rangle \\
& \geq\left\langle y_{t}-u_{n_{i}}, B y_{t}\right\rangle-\left\langle B x_{n_{i}}, y_{t}-u_{n_{i}}\right\rangle-\left\langle y_{t}-u_{n_{i}}, \frac{u_{n_{i}}-x_{n_{i}}}{s_{n_{i}}}\right\rangle+F\left(y_{t}, u_{n_{i}}\right) \\
& =\left\langle y_{t}-u_{n_{i}}, B y_{t}-B u_{n_{i}}\right\rangle+\left\langle y_{t}-u_{n_{i}}, B u_{n_{i}}-B x_{n_{i}}\right\rangle-\left\langle y_{t}-u_{n_{i}}, \frac{u_{n_{i}}-x_{n_{i}}}{s_{n_{i}}}\right\rangle \\
& \quad+F\left(y_{t}, u_{n_{i}}\right) .
\end{aligned}
$$

Since $B$ is monotone, we obtain from (A4) that $\left\langle y_{t}-w, B y_{t}\right\rangle \geq F\left(y_{t}, w\right)$. Using (A1) and (A4), we find that

$$
\begin{aligned}
0 & =F\left(y_{t}, y_{t}\right) \leq t F\left(y_{t}, y\right)+(1-t) F\left(y_{t}, w\right) \\
& \leq t F\left(y_{t}, y\right)+(1-t)\left\langle y_{t}-w, B y_{t}\right\rangle \\
& =t F\left(y_{t}, y\right)+(1-t) t\left\langle y-w, B y_{t}\right\rangle .
\end{aligned}
$$

Hence, $0 \leq F\left(y_{t}, y\right)+(1-t)\left\langle y-w, B y_{t}\right\rangle, \forall y \in C$. It follows from (A3) that $w \in \operatorname{EP}(F, B)$. Next, we prove that $q \in \bigcap_{i=1}^{\infty} F\left(S_{i}\right)$. Suppose to the contrary, $q \notin \bigcap_{i=1}^{\infty} F\left(S_{i}\right)$, i.e., $W q \neq q$. Since $y_{n_{i}} \rightarrow q$ and the space satisfies Opial's condition, one has

$$
\begin{aligned}
\liminf _{i \rightarrow \infty}\left\|y_{n_{i}}-q\right\| & <\liminf _{i \rightarrow \infty}\left\|y_{n_{i}}-W q\right\| \\
& \leq \liminf _{i \rightarrow \infty}\left\{\left\|y_{n_{i}}-W y_{n_{i}}\right\|+\left\|W y_{n_{i}}-W q\right\|\right\} \\
& \leq \liminf _{i \rightarrow \infty}\left\{\left\|y_{n_{i}}-W y_{n_{i}}\right\|+\left\|y_{n_{i}}-q\right\|\right\} .
\end{aligned}
$$


Since $\left\|W y_{n}-y_{n}\right\| \leq \sup _{x \in K}\left\|W x-W_{n} x\right\|+\left\|W_{n} y_{n}-y_{n}\right\|$, we find from Lemma 2.5 that $\lim _{n \rightarrow \infty}\left\|W y_{n}-y_{n}\right\|=0$. It follows that $\liminf _{i \rightarrow \infty}\left\|y_{n_{i}}-q\right\|<\liminf _{i \rightarrow \infty}\left\|y_{n_{i}}-q\right\|$. This leads to a contradiction. Thus, we have $q \in \bigcap_{i=1}^{\infty} F\left(S_{i}\right)$. This proves that $q \in F$. Therefore, one has

$$
\limsup _{n \rightarrow \infty}\left\langle f(\bar{x})-\bar{x}, x_{n}-\bar{x}\right\rangle \leq 0
$$

Finally, we show that $x_{n} \rightarrow \bar{x}$, as $n \rightarrow \infty$. Note that

$$
\begin{aligned}
\left\|x_{n+1}-\bar{x}\right\|^{2} & \\
\leq & \alpha_{n}\left\langle f\left(x_{n}\right)-f(\bar{x}), x_{n+1}-\bar{x}\right\rangle+\alpha_{n}\left\langle f(\bar{x})-\bar{x}, x_{n+1}-\bar{x}\right\rangle \\
& +\beta_{n}\left\|x_{n}-\bar{x}\right\|\left\|x_{n+1}-\bar{x}\right\|+\gamma_{n}\left\|y_{n}-\bar{x}\right\|\left\|x_{n+1}-\bar{x}\right\| \\
\leq & \frac{\kappa}{2} \alpha_{n}\left(\left\|x_{n}-\bar{x}\right\|^{2}+\left\|x_{n+1}-\bar{x}\right\|^{2}\right)+\alpha_{n}\left\langle f(\bar{x})-\bar{x}, x_{n+1}-\bar{x}\right\rangle \\
& +\left(1-\alpha_{n}\right)\left\|x_{n}-\bar{x}\right\|\left\|x_{n+1}-\bar{x}\right\| \\
\leq & \frac{1-\alpha_{n}(1-\kappa)}{2}\left\|x_{n}-\bar{x}\right\|^{2}+\frac{1}{2}\left\|x_{n+1}-\bar{x}\right\|^{2}+\alpha_{n}\left\langle f(\bar{x})-\bar{x}, x_{n+1}-\bar{x}\right\rangle,
\end{aligned}
$$

which implies that

$$
\left\|x_{n+1}-\bar{x}\right\|^{2} \leq\left(1-\alpha_{n}(1-\kappa)\right)\left\|x_{n}-\bar{x}\right\|^{2}+2 \alpha_{n}\left\langle f(\bar{x})-\bar{x}, x_{n+1}-\bar{x}\right\rangle .
$$

Using Lemma 2.1, we find that $\lim _{n \rightarrow \infty}\left\|x_{n}-\bar{x}\right\|=0$. This completes the proof.

\section{Applications}

Recall that a mapping $T: C \rightarrow C$ is said to be a $k$-strict pseudo-contraction if there exists a constant $k \in[0,1)$ such that

$$
\|T x-T y\|^{2} \leq\|x-y\|^{2}+k\|(I-T) x-(I-T) y\|^{2}
$$

for all $x, y \in C$. Note that the class of $k$-strict pseudo-contractions strictly includes the class of nonexpansive mappings. Put $A=I-T$, where $T: C \rightarrow C$ is a $k$-strict pseudocontraction. Then $A$ is $\frac{1-k}{2}$-inverse-strongly monotone. Now, we are in a position to state a results on fixed points of strict pseudo-contractions.

Theorem 4.1 Let $C$ be a nonempty closed convex subset of a Hilbert space $H$ and let $F$ be a bifunction from $C \times C$ to $\mathbb{R}$ which satisfies (A1)-(A4). Let $T: C \rightarrow H$ be a $k$-strict pseudo-contraction, $B: C \rightarrow H$ be a $\beta$-inverse-strongly monotone mapping, and $\left\{S_{i}: C \rightarrow C\right\}$ be a family of infinitely nonexpansive mappings. Assume that $F:=\bigcap_{i=1}^{\infty} F\left(S_{i}\right) \cap \operatorname{EP}(F, B) \cap F(T) \neq \emptyset$. Let $f: C \rightarrow C$ be a $\kappa$-contraction. Let $\left\{x_{n}\right\}$ be a sequence generated by $x_{1} \in C$ and

$$
\left\{\begin{array}{l}
F\left(u_{n}, y\right)+\left\langle B x_{n}, y-u_{n}\right\rangle+\frac{1}{s_{n}}\left\langle y-u_{n}, u_{n}-x_{n}\right\rangle \geq 0, \quad \forall y \in C, \\
y_{n}=\left(1-r_{n}\right) u_{n}+r_{n} T u_{n}, \\
x_{n+1}=\alpha_{n} f\left(x_{n}\right)+\beta_{n} x_{n}+\gamma_{n} W_{n} y_{n}, \quad \forall n \geq 1,
\end{array}\right.
$$


where $\left\{W_{n}: C \rightarrow C\right\}$ is the sequence generated in (2.4), $\left\{\alpha_{n}\right\},\left\{\beta_{n}\right\}$, and $\left\{\gamma_{n}\right\}$ are sequences in $(0,1)$ such that $\alpha_{n}+\beta_{n}+\gamma_{n}=1$ and $\left\{r_{n}\right\}$, and $\left\{s_{n}\right\}$ are positive number sequences. Assume that the above control sequences satisfy the following restrictions:

(a) $0<r \leq s_{n} \leq r^{\prime}<2 \beta, 0<r^{\prime \prime} \leq r_{n} \leq r^{\prime \prime \prime}<1-k$;

(b) $\lim _{n \rightarrow \infty} \alpha_{n}=0$ and $\sum_{n=1}^{\infty} \alpha_{n}=\infty$;

(c) $0<\liminf _{n \rightarrow \infty} \beta_{n} \leq \limsup \sup _{n \rightarrow \infty} \beta_{n}<1$;

(d) $\lim _{n \rightarrow \infty}\left|s_{n}-s_{n+1}\right|=\lim _{n \rightarrow \infty}\left|r_{n}-r_{n+1}\right|=0$.

Then the sequence $\left\{x_{n}\right\}$ converges strongly to $\bar{x}=\operatorname{Proj}_{F} f(\bar{x})$.

Proof Taking $A=I-T$, wee see that $A: C \rightarrow H$ is a $\alpha$-strict pseudo-contraction with $\alpha=\frac{1-k}{2}$ and $F(T)=\operatorname{VI}(C, A)$. Using Theorem 3.1, we find the desired conclusion immediately.

Let $g: H \rightarrow(-\infty,+\infty]$ be a proper convex lower semi-continuous function. Then the subdifferential $\partial g$ of $g$ is defined as follows:

$$
\partial f g(x)=\{y \in H: g(z) \geq g(x)+\langle z-x, y\rangle, z \in H\}, \quad \forall x \in H .
$$

From Rockafellar [30], we know that $\partial g$ is maximal monotone. It is not hard to verify that $0 \in \partial g(x)$ if and only if $g(x)=\min _{y \in H} g(y)$.

Let $I_{C}$ be the indicator function of $C$, i.e.,

$$
I_{C}(x)= \begin{cases}0, & x \in C, \\ +\infty, & x \notin C .\end{cases}
$$

Since $I_{C}$ is a proper lower semi-continuous convex function on $H$, we see that the subdifferential $\partial I_{C}$ of $I_{C}$ is a maximal monotone operator. It is clear that $J_{r} x=P_{C} x, \forall x \in H$. Notice that $\left(A+\partial I_{C}\right)^{-1}(0)=\mathrm{VI}\left(C, A_{1}\right)$. Now, we are in a position to state the result on variational inequalities.

Theorem 4.2 Let $C$ be a nonempty closed convex subset of a Hilbert space $H$ and let $F$ be a bifunction from $C \times C$ to $\mathbb{R}$ which satisfies (A1)-(A4). Let $A: C \rightarrow H$ be an $\alpha$-inversestrongly monotone mapping, $B: C \rightarrow H$ be a $\beta$-inverse-strongly monotone mapping, and $\left\{S_{i}: C \rightarrow C\right\}$ be a family of infinitely nonexpansive mappings. Assume that $F:=\bigcap_{i=1}^{\infty} F\left(S_{i}\right) \cap$ $\operatorname{EP}(F, B) \cap \operatorname{VI}(C, A) \neq \emptyset$. Let $f: C \rightarrow C$ be a $\kappa$-contraction. Let $\left\{x_{n}\right\}$ be a sequence generated by $x_{1} \in C$ and

$$
\left\{\begin{array}{l}
F\left(u_{n}, y\right)+\left\langle B x_{n}, y-u_{n}\right\rangle+\frac{1}{s_{n}}\left\langle y-u_{n}, u_{n}-x_{n}\right\rangle \geq 0, \quad \forall y \in C, \\
x_{n+1}=\alpha_{n} f\left(x_{n}\right)+\beta_{n} x_{n}+\gamma_{n} W_{n} P_{C}\left(u_{n}-s_{n} A u_{n}\right), \quad \forall n \geq 1,
\end{array}\right.
$$

where $\left\{W_{n}: C \rightarrow C\right\}$ is the sequence generated in (2.4), $\left\{\alpha_{n}\right\},\left\{\beta_{n}\right\}$, and $\left\{\gamma_{n}\right\}$ are sequences in $(0,1)$ such that $\alpha_{n}+\beta_{n}+\gamma_{n}=1$ and $\left\{r_{n}\right\}$, and $\left\{s_{n}\right\}$ are positive number sequences. Assume that the above control sequences satisfy the following restrictions:

(a) $0<r \leq s_{n} \leq r^{\prime}<2 \beta, 0<r^{\prime \prime} \leq r_{n} \leq r^{\prime \prime \prime}<2 \alpha$;

(b) $\lim _{n \rightarrow \infty} \alpha_{n}=0$ and $\sum_{n=1}^{\infty} \alpha_{n}=\infty$; 
(c) $0<\liminf _{n \rightarrow \infty} \beta_{n} \leq \limsup _{n \rightarrow \infty} \beta_{n}<1$;

(d) $\lim _{n \rightarrow \infty}\left|s_{n}-s_{n+1}\right|=\lim _{n \rightarrow \infty}\left|r_{n}-r_{n+1}\right|=0$.

Then the sequence $\left\{x_{n}\right\}$ converges strongly to $\bar{x}=\operatorname{Proj}_{F} f(\bar{x})$.

\section{Competing interests}

The author declares to have no competing interests.

\section{Acknowledgements}

The author is grateful to Prof. Tian Cheng and the reviewers' suggestions which improved the contents of the article.

Received: 5 May 2014 Accepted: 16 July 2014 Published: 21 August 2014

\section{References}

1. Hao, Y: On variational inclusion and common fixed point problems in Hilbert spaces with applications. Appl. Math. Comput. 217, 3000-3010 (2010)

2. Zegeye, $\mathrm{H}$, Shahzad, N: Strong convergence theorem for a common point of solution of variational inequality and fixed point problem. Adv. Fixed Point Theory 2, 374-397 (2012)

3. Wu, C, Liu, A: Strong convergence of a hybrid projection iterative algorithm for common solutions of operator equations and of inclusion problems. Fixed Point Theory Appl. 2012, Article ID 90 (2012)

4. Wang, ZM, Lou, W: A new iterative algorithm of common solutions to quasi-variational inclusion and fixed point problems. J. Math. Comput. Sci. 3, 57-72 (2013)

5. Cho, SY, Qin, X, Wang, L: Strong convergence of a splitting algorithm for treating monotone operators. Fixed Point Theory Appl. 2014, Article ID 94 (2014)

6. LV, S: Strong convergence of a general iterative algorithm in Hilbert spaces. J. Inequal. Appl. 2013, Article ID 19 (2013)

7. Wang, $\mathrm{Y}, \mathrm{LuO}, \mathrm{H}$ : Iterative approximation for the common solutions of a infinite variational inequality system for inverse-strongly accretive mappings. J. Math. Comput. Sci. 2, 1660-1670 (2012)

8. Cho, SY, Li, W, Kang, SM: Convergence analysis of an iterative algorithm for monotone operators. J. Inequal. Appl. 2013, Article ID 199 (2013)

9. Cho, SY, Kang, SM: Approximation of common solutions of variational inequalities via strict pseudocontractions. Acta Math. Sci. 32, 1607-1618 (2012)

10. liduka, $\mathrm{H}$ : Strong convergence for an iterative method for the triple-hierarchical constrained optimization problem. Nonlinear Anal. 71, e1292-e1297 (2009)

11. Qin, X, Shang, M, Su, Y: Strong convergence of a general iterative algorithm for equilibrium problems and variational inequality problems. Math. Comput. Model. 48, 1033-1046 (2008)

12. Wang, S, Zhou, C: New iterative scheme for finite families of equilibrium, variational inequality, and fixed point problems in Banach spaces. Fixed Point Theory Appl. 2011, Article ID 372975 (2011)

13. Yu, L, Song, J: Strong convergence theorems for solutions of fixed point and variational inequality problems. J. Inequal. Appl. 2014, Article ID 215 (2014)

14. Rodjanadid, B, Sompong, S: A new iterative method for solving a system of generalized equilibrium problems, generalized mixed equilibrium problems and common fixed point problems in Hilbert spaces. Adv. Fixed Point Theory 3, 675-707 (2013)

15. Kim, JK: Strong convergence theorems by hybrid projection methods for equilibrium problems and fixed point problems of the asymptotically quasi- $\boldsymbol{\phi}$-nonexpansive mappings. Fixed Point Theory Appl. 2011, Article ID 10 (2011)

16. Kim, JK, Anh, PN, Nam, YM: Strong convergence of an extended extragradient method for equilibrium problems and fixed point problems. J. Korean Math. Soc. 49, 187-200 (2012)

17. Kim, JK, Buong, N: A new iterative method for equilibrium problems and fixed point problems for infinite family of nonself strictly pseudocontractive mappings. Fixed Point Theory Appl. 2013, Article ID 286 (2013)

18. Qin, X, Cho, SY, Kang, SM: An extragradient-type method for generalized equilibrium problems involving strictly pseudocontractive mappings. J. Glob. Optim. 49, 679-693 (2011)

19. Zhang, M: An algorithm for treating asymptotically strict pseudocontractions and monotone operators. Fixed Point Theory Appl. 2014, Article ID 52 (2014)

20. Song, J, Chen, M: On generalized asymptotically quasi- $\phi$-nonexpansive mappings and a Ky Fan inequality. Fixed Point Theory Appl. 2013, Article ID 237 (2013)

21. Qin, X, Cho, SY, Kang, SM: Iterative algorithms for variational inequality and equilibrium problems with applications. J. Glob. Optim. 48, 423-445 (2010)

22. Cho, SY, Qin, X: On the strong convergence of an iterative process for asymptotically strict pseudocontractions and equilibrium problems. Appl. Math. Comput. 235, 430-438 (2014)

23. Takahashi, W, Yao, JC: Nonlinear operators of monotone type and convergence theorems with equilibrium problems in Banach spaces. Taiwan. J. Math. 15, 787-818 (2011)

24. Qin, X, Cho, SY, Kang, SM: Some results on variational inequalities and generalized equilibrium problems with applications. Comput. Appl. Math. 29, 393-421 (2010)

25. Chang, SS, Lee, HWJ, Chan, CK: A new method for solving equilibrium problem fixed point problem and variational inequality problem with application to optimization. Nonlinear Anal. 70, 3307-3319 (2009)

26. Liu, LS: Ishikawa and Mann iteration process with errors for nonlinear strongly accretive mappings in Banach spaces. J. Math. Anal. Appl. 194, 114-125 (1995)

27. Blum, E, Oettli, W: From optimization and variational inequalities to equilibrium problems. Math. Stud. 63, 123-145 (1994) 
28. Shimoji, K, Takahashi, W: Strong convergence to common fixed points of infinite nonexpansive mappings and applications. Taiwan. J. Math. 5, 387-404 (2001)

29. Cho, SY: Strong convergence of an iterative algorithm for sums of two monotone operators. J. Fixed Point Theory 2013, Article ID 6 (2013)

30. Rockafellar, RT: On the maximality of sums of nonlinear monotone operators. Trans. Am. Math. Soc. 149, 75-88 (1970)

31. Suzuki, T: Strong convergence of Krasnoselskii and Mann's type sequences for one-parameter nonexpansive semigroups without Bochne integrals. J. Math. Anal. Appl. 305, 227-239 (2005)

doi:10.1186/1029-242X-2014-313

Cite this article as: Zhang: Strong convergence of a Halpern-type algorithm for common solutions of fixed point and equilibrium problems. Journal of Inequalities and Applications 2014 2014:313.

\section{Submit your manuscript to a SpringerOpen ${ }^{\circ}$} journal and benefit from:

- Convenient online submission

Rigorous peer review

- Immediate publication on acceptance

- Open access: articles freely available online

- High visibility within the field

- Retaining the copyright to your article 